\title{
Search for energy dependent patterns in the arrival direction of cosmic rays at the Pierre Auger Observatory
}

Tobias Winchen $^{* a}$ for the Pierre Auger Collaboration ${ }^{b}$

${ }^{a}$ Bergische Universität Wuppertal, Department of Physics, 42119 Wuppertal, Germany

${ }^{b}$ Observatorio Pierre Auger, Av. San Martín Norte 304, 5613 Malargüe, Argentina

E-mail: auger_spokespersons@fnal.gov

Full author list: http://www.auger.org/archive/authors_2015_06.html

\begin{abstract}
Energy-dependent patterns in the arrival directions of cosmic rays are expected from deflections in galactic and extragalactic magnetic fields. We report on searches for such patterns in the data of the surface detector of the Pierre Auger Observatory at energies above $E=5 \mathrm{EeV}$ in regions within approximately $15^{\circ}$ around events with energy $E>60 \mathrm{EeV}$. No significant patterns are found with this analysis which can be used to constrain parameters in propagation scenarios.
\end{abstract}

The 34th International Cosmic Ray Conference,

30 July- 6 August, 2015

The Hague, The Netherlands

\footnotetext{
* Speaker.
} 


\section{Introduction}

The sources of ultra-high energy cosmic rays (UHECR) have not been identified so far, presumably because of the deflection of the charged cosmic rays in the galactic and extragalactic magnetic fields. However, from such deflections the distribution of UHECRs arrival directions may show energy-dependent patterns. In particular, a circular 'blurring' of the sources is expected from deflection in turbulent magnetic fields, while energy-dependent linear structures are expected from deflection in coherent magnetic fields. To search for such patterns in the data collected with the Pierre Auger Observatory [1], we investigate the local Regions Of Interest (ROI) around cosmic rays with $E \geq 60 \mathrm{EeV}$. We analyzed the cosmic rays with energies above $E=5 \mathrm{EeV}$ arriving within an angular separation of $0.25 \mathrm{rad}$ using two independent methods [2]. In one method we study energy-energy correlations between pairs of cosmic rays depending on their angular separation from the center of the region. This method is sensitive to the circular patterns expected from particle deflection in turbulent magnetic fields $[3,4]$. In the second method we decompose the directional energy distribution of the cosmic rays along its principal axes. This method is sensitive to clusters of cosmic rays as well as to linear patterns expected from deflections in coherent magnetic fields $[5,6]$.

\section{Methods}

\subsection{Energy-Energy Correlations}

The Energy-energy correlation $\Omega_{i j}$ is calculated for every pair of UHECRs $i j$ within a ROI using

$$
\Omega_{i j}=\frac{\left(E_{i}-\left\langle E\left(\alpha_{i}\right)\right\rangle\right)\left(E_{j}-\left\langle E\left(\alpha_{j}\right)\right\rangle\right)}{E_{i} E_{j}}
$$

where $E_{i}$ is the energy of the UHECR $i$ with the angular separation $\alpha_{i}$ to the center of the ROI and $\left\langle E_{i}\left(\alpha_{i}\right)\right\rangle$ is the average energy of all UHECRs at the angular separation $\alpha_{i}$ from the center of the ROI. A pair of cosmic rays $i j$ can contribute positively or negatively to the distribution of $\Omega_{i j}$. If one particle has an energy above the corresponding average energy and the other below the corresponding average energy, the contribution is negative. A pair with both energies being below average as well as a pair with both being above average contribute positively. If there is a pattern in the energy distribution there are more positive correlations than expected from isotropically distributed cosmic rays.

\subsection{Principal Axes}

The system of principal axes of the energy distribution $\vec{n}_{k=1,2,3}$ is calculated by successive maximization of the quantity

$$
T_{k}=\max _{\vec{n}_{k}}\left(\frac{\sum_{i}\left|\omega_{i}^{-1} \vec{p}_{i} \cdot \vec{n}_{k}\right|}{\sum_{i}\left|\omega_{i}^{-1} \vec{p}_{i}\right|}\right)
$$

with respect to the axes $\vec{n}_{k}$ starting with $k=1$. Here $\vec{p}_{i}$ is the momentum and $\omega_{i}$ the exposure of the detector [7] in the direction of particle $i$. The resulting values of $T_{k=1,2,3}$ quantify the strength of the collimation of the particle momenta along each of the three axes $\vec{n}_{k=1,2,3}$ of the principal system. 

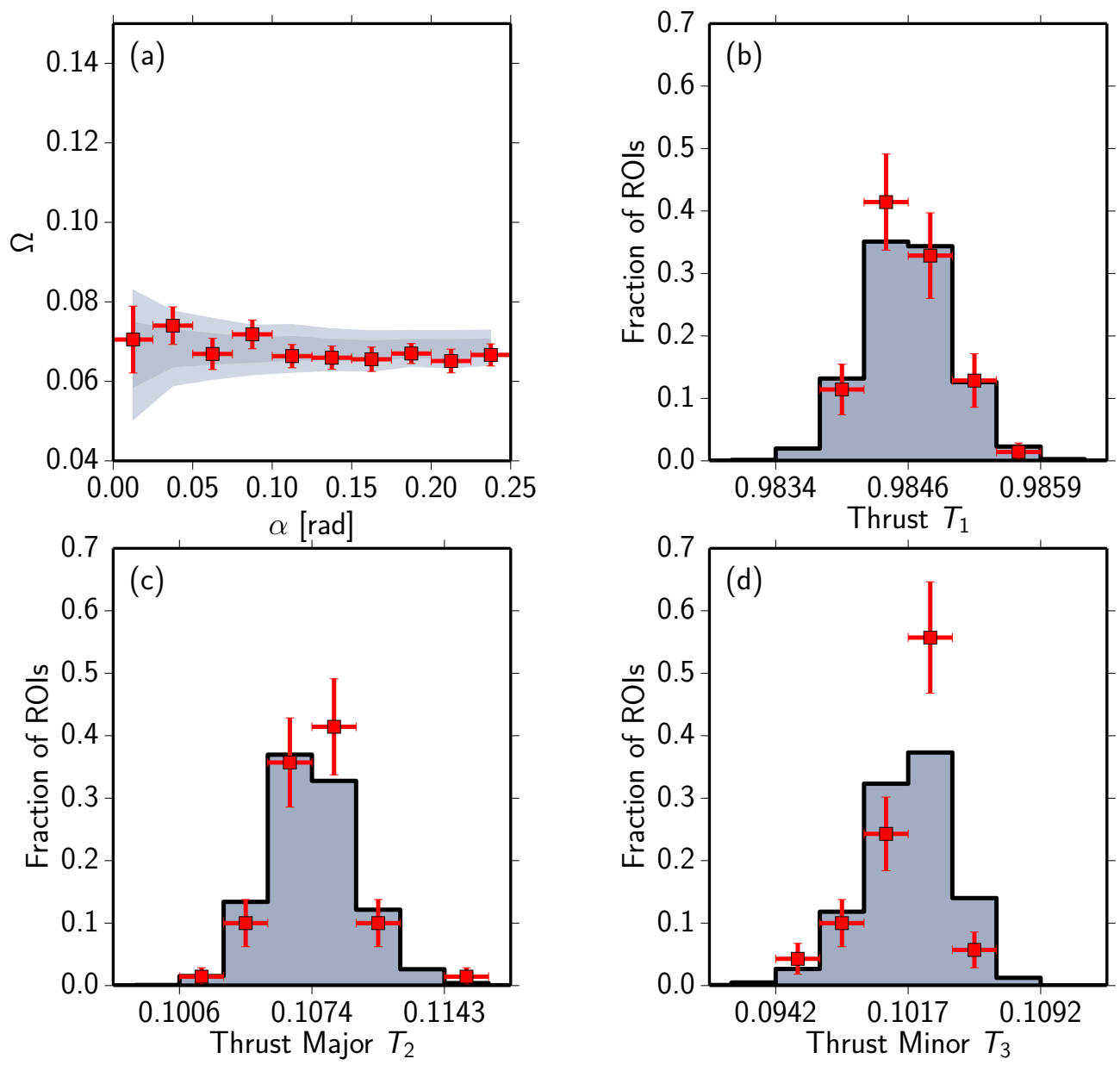

Figure 1: Measurement of the (a) energy-energy correlation $\Omega$ and (b-d) observables $T_{1,2,3}$ with the Pierre Auger Observatory (red squares and error bars). The measurements are compared to distributions without patterns in the arrival directions of UHECRs (filled distributions).

The first principal axis $\vec{n}_{1}$ is the radial unit vector $\vec{e}_{r}$ pointing to the local barycenter of the energy distribution. The $T_{1}$ value is thus a measure for the energy-weighted strength of clustering of the events. It is $T_{1}=1$ for no dispersion of the particles in the region, whereas for an isotropic distribution of UHECRs in a region the expectation value is determined by the size of the region [6].

The second and third principal axes $\vec{n}_{2}$ and $\vec{n}_{3}$ can be written as linear combination of the unit vectors $\vec{e}_{\phi}$ and $\vec{e}_{\theta}$. The $T_{2}$ value becomes maximal if $\vec{n}_{2}$ is aligned with a linear distribution of UHECR arrival directions and can be thus used as a generalized multiplet analysis. It thus points along threadlike structures in the energy distribution of UHECRs. As the $\vec{n}_{2}$ axis is chosen perpendicular to $\vec{n}_{1}$ and $\vec{n}_{2}$ it has no additional physical meaning. However, the $T_{3}$ value contains information as it denotes the collimation strength perpendicular to the $\vec{n}_{2}$ axis.

\section{Results}

We measured the EEC and $T_{1,2,3}$ distributions using 30,664 events recorded with the surface 
detector of the Pierre Auger Observatory above $5 \mathrm{EeV}$. For the selected events we further required that the zenith angle of the events is smaller than $60^{\circ}$ and that the detector stations surrounding the station with the highest signal are active [8] to obtain a sample with minimum potential biases. Of the selected events, 70 have an energy $E \geq 60 \mathrm{EeV}$ and are at least $0.25 \mathrm{rad}$ inside the field of view of the Pierre Auger Observatory. These events mark the ROIs used in this analysis.

In Figure 1 the distributions of the EEC and the $T_{1,2,3}$ observables are shown together with the distributions expected from isotropic arrival directions of UHECRs. The measured distributions of all four observables reveal no local patterns in the arrival directions of UHECRs. The goodness-of-fit of the measurements compared to expected distributions without structure in the arrival directions of UHECRs using a $\chi^{2}$ test, yields $p$-values which are all above $p=0.2$ except for the $T_{3}$ distribution with $p\left(T_{3}\right)=0.01$. However, this low $p$-value results from a lack of signallike regions in the data which are expected to broaden the distribution, and thus does not indicate significant patterns.

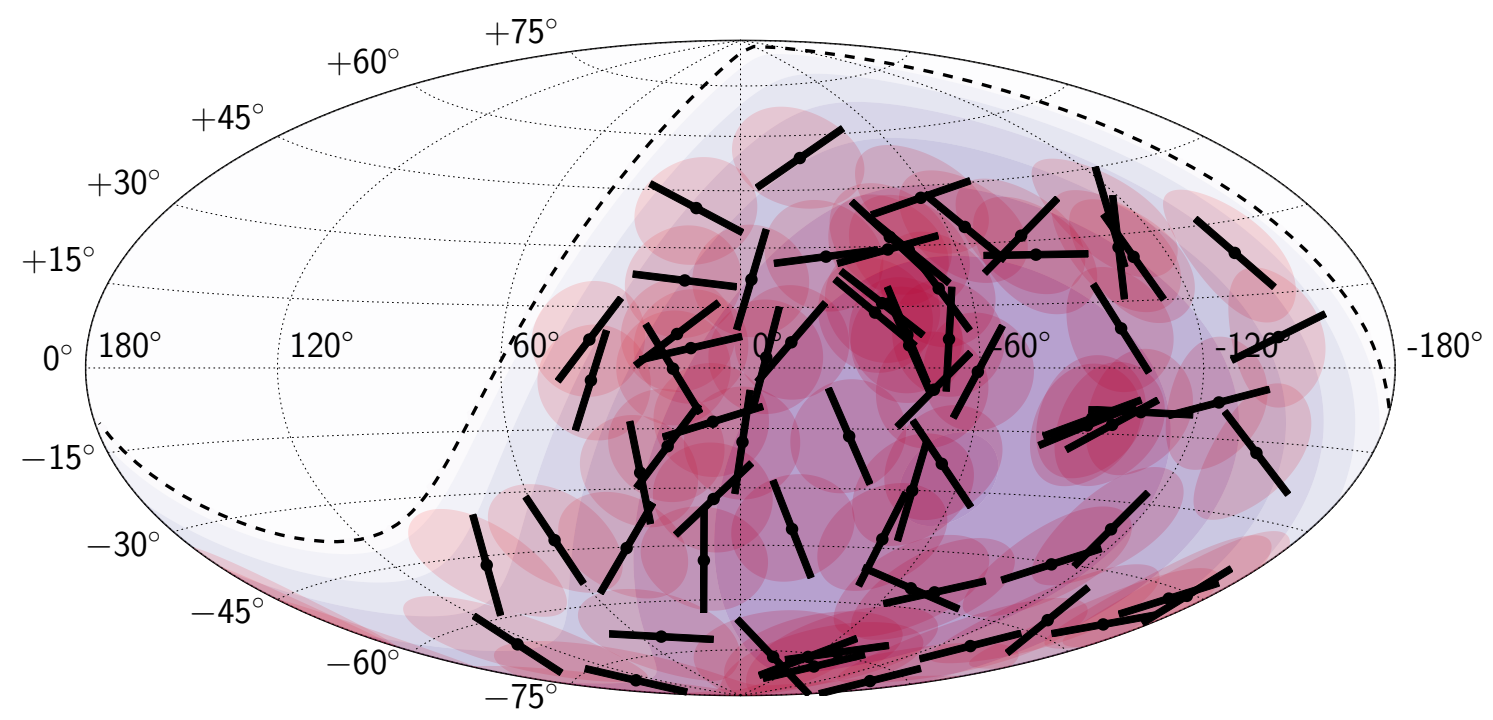

Figure 2: Map of principal axes of the directional energy distribution using a Hammer projection and galactic coordinates. The red shaded areas represent the regions of interest. Black lines denote the second principal axes $\vec{n}_{2}$ and black dots mark the positions of axes $\vec{n}_{1}$. The blue shading indicates the exposure of the Pierre Auger Observatory; the dashed line marks the extent of its field of view.

In addition to the scalar distributions, the direction of the second principal axes $\vec{n}_{2}$ can be displayed as the map shown in figure 2. If the axes are non-trivial, this map displays the directions of deflection of UEHCR in coherent cosmic magnetic fields. In simulation studies it was shown that the distribution of principal axes can contain information, even though the $T_{1,2,3}$ scalar values are compatible with isotropy [6]. We tested the reproducibility of the axes in subsets of the data by splitting the dataset into 12 independent subsets by chance and analyzing the variance of the directions in the subsets. We found no reproducibility of the axes in this analysis. 


\section{Discussion and Conclusions}

The non observation of significant patterns can be used to exclude all scenarios that predict otherwise. To illustrate the required procedure we simulated a simple model for extragalactic propagation of protons based on parameterizations as implemented in version 1.2 of the PARSEC software [9]. In particular we assume here, that the UHECRs originate from isotropically distributed point sources with equal luminosity and that the root-mean-square of the deflections of UHECRs with energies $E$ from a source in distance $D$ can be parametrized as $\delta_{\mathrm{RMS}}=C_{\mathrm{E}} \frac{\sqrt{D}}{E}$. The calculations further account for energy losses of the UHECRs from interaction with extragalactic-photon backgrounds, effects from the expansion of the universe, and the deflection in the galactic magnetic field using the model proposed by Jansson and Farrar [10, 11].

We scanned the density of point sources and the strength of the deflection in the extragalactic magnetic field $C_{\mathrm{E}}$ in this scenario and derived a combined limit on both parameters using the $C L_{S}$ method [12]. Within this simplified scenario, we found that the deflection in the extragalactic magnetic field has to be larger than $C_{\mathrm{E}}=10-120^{\circ} \mathrm{Mpc}^{-1 / 2} \mathrm{EeV}$ for source densities smaller than $10^{-3} \mathrm{Mpc}^{-3}$. For protons with an energy $E=10 \mathrm{EeV}$ from a source at $16 \mathrm{Mpc}$ this translates to a required strength of the deflection in extragalactic space of more than $4^{\circ}$ if the source density is smaller than $10^{-3} \mathrm{Mpc}^{-3}$ and more than $25^{\circ}$ if the source density is smaller than $10^{-4} \mathrm{Mpc}^{-3}$.

\section{References}

[1] The Pierre Auger Collaboration, The Pierre Auger Cosmic Ray Observatory, Accepted for publication in Nuclear Instruments and Methods (NIM) A (2015) [arXiv: 1502.01323 ].

[2] Pierre Auger Collaboration, A. Aab et al., Search for patterns by combining cosmic-ray energy and arrival directions at the Pierre Auger Observatory, European Physical Journal C (EPJ C) 75 (2015) 269, [arXiv: 1410 . 0515].

[3] M. Erdmann and P. Schiffer, A Method of Measuring Cosmic Magnetic Fields with Ultra High Energy Cosmic Ray Data, Astroparticle Physics 33 (2010) 201-205, [arXiv:0904.4888].

[4] P. Schiffer, Constraining Cosmic Magnetic Fields by a Measurement of Energy-EnergyCorrelations with the Pierre Auger Observatory. PhD thesis, RWTH Aachen University, 2011.

[5] M. Erdmann and T. Winchen, Detecting Local Deflection Patterns of Ultra-high Energy Cosmic Rays using the Principal Axes of the Directional Energy Distribution, in Proceedings of the 33rd ICRC, 2013. [arXiv:1307.8273].

[6] T. Winchen, The Principal Axes of the Directional Energy Distribution of Cosmic Rays Measured with the Pierre Auger Observatory. PhD thesis, RWTH Aachen University, 2013.

[7] P. Sommers, Cosmic Ray Anisotropy Analysis with a Full-Sky Observatory, Astroparticle Physics 14 (2001) 271-286, [arXiv: astro-ph/ 0004016 ]. 
[8] Pierre Auger Collaboration, J. Abraham et al., Trigger and aperture of the surface detector array of the Pierre Auger Observatory, Nuclear Instruments and Methods (NIM) $\mathbf{A 6 1 3}$ (2010) 29-39, [arXiv:1111.6764].

[9] H.-P. Bretz, M. Erdmann, P. Schiffer, D. Walz, and T. Winchen, PARSEC: A Parametrized Simulation Engine for Ultra-High Energy Cosmic Ray Protons, Astroparticle Physics 54 (2014) 110-117, [arXiv:1302.3761].

[10] R. Jansson and G. R. Farrar, A New Model of the Galactic Magnetic Field, Astrophysical Journal 757 (2012) 14, [arXiv: 1204 .3662].

[11] R. Jansson and G. R. Farrar, The Galactic Magnetic Field, Astrophysical Journal 761 (2012) L11, [arXiv:1210.7820].

[12] A. L. Read, Presentation of search results: the $C L_{s}$ technique, Journal of Physics $G \mathbf{2 8}$ (2002) 2693-2704. 